\title{
MODE-COUPLING BEHAVIOUR OF THE GENERALIZED HYDRODYNAMIC MODES FOR A LENNARD-JONES FLUID
}

\author{
I. M. Mryglod \\ Institute for Condensed Matter Physics of National Academy of Sciences of Ukraine, \\ 1 Svientsitskii Str., Lviv, UA-290011, Ukraine
}

(Received September 7, 1998; received in final form November 16, 1998)

\begin{abstract}
The mode-coupling effects, observed in the behaviour of generalized hydrodynamic modes, are analyzed. The spectrum of collective modes has been calculated [Phys. Lett. A 205 (1995) 401] in nine-mode approximation of the generalized collective mode approach for a Lennard-Jones fluid. It is shown that the obtained results are in full agreement with the predictions of the modecoupling theory. The mode-coupling parameters, describing the non-analytic contributions to the generalized hydrodynamic modes spectrum, are calculated. We discuss also the relation between both the theories considered.
\end{abstract}

Key words: Generalized hydrodynamic modes, mode-coupling theory, Lennard-Jones fluid.

PACS numbers: 05.20.-y, 05.60.+w, 05.70.Ln

\section{INTRODUCTION}

Generalized hydrodynamics of fluids is still one of the most attractive fields of modern theory of fluids. In the last twenty years a lot of attempts to bridge the linear Navier-Stocks hydrodynamics and the kinetic theory have been made $[1,2]$, but many issues remain unresolved.

Recently, the concept of generalized collective modes (GCM) for the description of dense fluid systems has been developed [3-9]. In this approach the dynamics of dense gases and liquids can be described in terms of the so-called generalized collective modes. Some of them are an extension of the hydrodynamic modes known from the Navier-Stocks hydrodynamic treatment, and the other ones are the generalized kinetic collective modes which reflex the short-time behaviour related to molecular as well as kinetic regimes. One of the most significant advantages of the GCM approach is the possibility to describe consistently the dynamic properties of fluids in a wide range of wavenumbers $k$ and frequencies $\omega$, starting from the hydrodynamic values and up to the Gaussianlike (free-particle) limit (see also [10]) that is in fact the main test for any theory to be considered as the generalized hydrodynamic theory. One additional aspect, not studied as yet, is a relation of the GCM approach to the mode-coupling (MC) theories [11-18], which were very successful in the explanation for long-time tails of timecorrelation functions as well as for non-analytic dispersions of the generalized transport coefficients and the generalized hydrodynamic modes for small wavenumbers $k$ and frequencies $\omega$. The goal of our study is to analyze this problem in more detail, using our previous results obtained for the generalized collective mode spectra of a Lennard-.Jones fluid $[5,6]$ in five-, seven-, and ninemode approximations of the GCM approach. A special attention is paid on the behaviour of generalized hydrodynamic modes at small and intermediate values $k$.

\section{GENERALIZED HYDRODYNAMIC MODES}

According to the predictions of the mode-coupling theory, for small wavenumbers $k$ the generalized hydrodynamic modes behave as follows $[12,16,15]$ :

(i) the generalized heat mode

$$
\begin{aligned}
z_{h}(k) & =\sigma_{h}(k), \\
\sigma_{h}(k) & =D k^{2}-a_{h} k^{5 / 2}+O\left(k^{11 / 4}\right) ;
\end{aligned}
$$

(ii) the generalized sound modes

$$
z_{s}^{ \pm}(k)= \pm \omega_{s}(k)+\sigma_{s}(k)
$$

with

$$
\begin{aligned}
& \omega_{s}(k)=c_{s} k+a_{s} k^{5 / 2}+O\left(k^{11 / 4}\right), \\
& \sigma_{s}(k)=\Gamma k^{2}-a_{s} k^{5 / 2}+O\left(k^{11 / 4}\right),
\end{aligned}
$$

where

$$
\begin{aligned}
& D=\frac{\lambda}{n c_{P}}, \quad c_{s}=\left(\frac{\gamma k_{B} T}{m S(0)}\right)^{1 / 2} \\
& \Gamma=\frac{1}{2}\left(\frac{\eta^{l}}{\rho}+(\gamma-1) D\right)
\end{aligned}
$$

are the thermal diffusivity, adiabatic sound velocity and the sound damping coefficient, respectively. Here $n$ and $\rho=m n$ denote the number and mass densities, $\gamma=$ $c_{P} / c_{V}$ is the ratio of specific heats at constant pressure 
and constant volume, $S(0)$ is a value of static structure factor at $k=0, \lambda$ and $\eta^{l}=4 \eta / 3+\zeta$ denote a thermal conductivity and longitudinal viscosity of a fluid. The first terms in Eqs. (1), (3) and (4) are the well-known hydrodynamic contributions which result from the NavierStocks hydrodynamics.

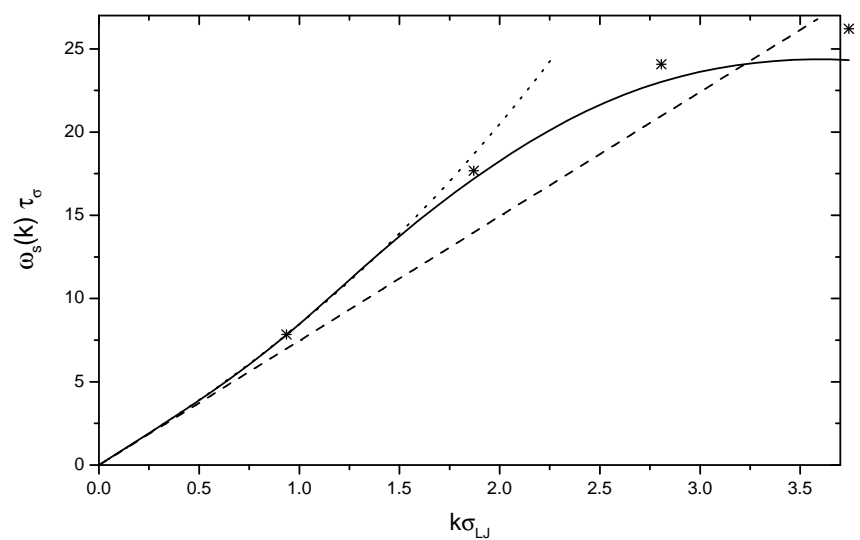

Fig. 1. Dispersion of generalized sound modes: the results of nine-mode approximation (solid curve), hydrodynamic behaviour (dashed line), mode-coupling prediction (dotted curve) and the results of five-mode approximation for $k / k_{\min }=1,2,3,4$ (stars), respectively.

The mode-coupling parameters $a_{s}$ and $a_{h}$ are expressed via the thermodynamic quantities and their derivatives. For instance, one has $[12,16,15]$

$$
a_{h}=\frac{1}{56 \pi} \frac{k_{B} T \sqrt{c_{s}}}{n m} \frac{\gamma-1}{\left(\alpha_{T} T\right)^{2}} \Gamma^{-3 / 2},
$$

where $\alpha_{T}$ is the thermal expansion coefficient. The MC expression for $a_{s}$ is more complex $[12,16,15]$ and involves the density derivative of $c_{s}$ and the temperature derivatives of $c_{P}$ and $\alpha_{T}$ well. It is more important for our consideration that both the coefficients $a_{s}$ and $a_{h}$ are usually positive for fluids, so that the equations, presented above, state that the dispersion of generalized sound modes has to be above the hydrodynamic result $c_{s} k$ and the damping coefficients of both generalized sound and generalized heat modes are below the hydrodynamic values when $k$ increases starting from the hydrodynamic values. Keeping these statements in mind let us analyze now the results obtained for the generalized collective mode spectrum of a Lennard--Jones fluid in Ref. $[5,6]$. The calculation has been performed at the reduced density $n^{*}=n \sigma_{L J}^{3}=0.845$ and the reduced temperature $T^{*}=k_{B} T / \epsilon_{L J}=1.705$ [20] within the five-, seven- and nine-mode approximations of GCM approach. We have used in our study the molecular dynamic (MD) data for the static correlation functions and the correlation times which were used in fact as the input parameters of the theory, so that the theory does not contain any adjustable parameters. In Ref. [6] for the study of the generalized collective modes spectrum within nine-mode approximation the additional interpo- lation procedure has been applied for the input parameters of the theory at intermediate values of $k$ located in between the MD points $k_{l}=l k_{\min }$ and $k_{l+1}=(l+1) k_{\min }$, where $l=0,1,2, \ldots, k_{\min }=2 \pi / L=0.936 / \sigma_{L J}$, and $L$ is the length of the simulation box $\left(L^{3}\right)$ considered. The values of the input parameters at $k=0$ have been found by extrapolating the MD data for $k \rightarrow 0$. Hence, one has a detailed structure of the generalized hydrodynamic modes which can be used for the next study of mode-coupling effects.

From the MD simulations (by extrapolating the MD results for the limit $k \rightarrow 0$ ) the following values for the thermodynamic quantities and the transport coefficients have been obtained [5,9]: $S(0)=0.05, \gamma=1.68$, $\alpha_{T} T=0.29, c_{V} / k_{B}=2.35, \lambda=7.71, \eta=2.37, \zeta=0.69$ $\left(\eta^{l}=3.85\right)$. All the quantities are given in dimensionless form using for the space, temperature and time scales the parameters of a Lennard-Jones potential, namely, $\sigma_{L J}$, $\epsilon_{L J}$, and $\tau_{\sigma}=\sigma_{L J}\left(m / \epsilon_{L J}\right)^{1 / 2}$, respectively. Hence, using the expressions given above we get $c_{s}=7.47, D=2.31$, $\Gamma=3.07, \Gamma / D=1.33$, and $a_{h}=0.05$.
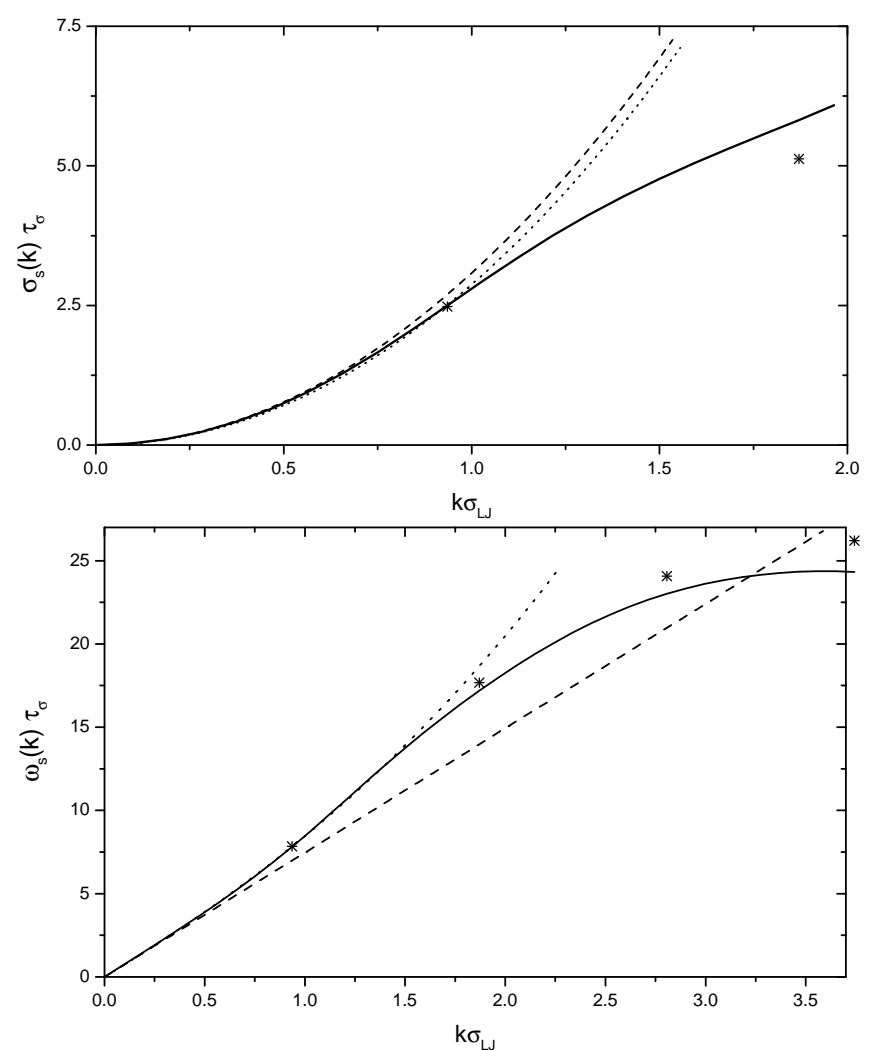

Fig. 2. Damping coefficient of generalized sound modes: the results of nine-mode approximation (solid curve), hydrodynamic behaviour (dashed line), mode-coupling prediction (dotted curve), and the results of five-mode approximation for $k / k_{\min }=1,2$ (stars), respectively.

In figure 1 we plot the dispersion of the generalized sound modes (solid curve). It is obvious that $\omega_{s}(k)$ is above the hydrodynamic result (showed by dashed line) up to $k \sigma_{L J}=3.3$ when $k$ increases from the hydrodynamic values. For the estimation of the MC parameter 
$a_{s}$ we used the fitting procedure for small values of $k$ (up to $k \sigma_{L J}=1.3$ ) and found that $a_{s}=0.98$. This result correlates very well with the result obtained in Ref. [19] within the MC treatment $\left(a_{s}=0.94\right)$. The MC behaviour of $\omega_{s}(k)$ (see $(3)$ ) is shown in figure 1 by a dotted curve.

In figures 2 and 3 the generalized damping coefficients for the sound and heat modes are plotted, respectively. One can see that both coefficients are below the hydrodynamic values shown by dashed curves. Hence, one may conclude that the results for the generalized hydrodynamic modes are in qualitative agreement with the mode coupling predictions. Let us analyze in more detail these results and try to estimate the MC parameters. It is important for the next consideration to mention that in Ref. [19] it was found that the first MC corrections related with the parameters $a_{s}$ and $a_{h}$ in Eqs. (1), (3) and (4) could be applied for the thermodynamic point considered in an extremely small range of $k$, so that the higher order contributions become significant for $k \sigma_{L J}>0.5$. In our study we observed that the most sensitive in this connection is the sound damping coefficient and the mode-coupling expression (4) describes correctly the behaviour of generalized sound modes for larger values $k$ only when the term proportional to $k^{11 / 4}$ is taken into account. Hence, we have used the following expression

$$
\sigma_{s}^{a}(k)=\Gamma k^{2}-a_{s} k^{5 / 2}+b_{s} k^{11 / 4},
$$

for fitting the MC prediction to the GCM results, where $b_{s}$ was considered as an adjustable parameter (the value $a_{s}$ has been found above). In such a manner, we obtained $b_{s}=0.78$, so that expression ( 7 ) can be then applied up to $k \sigma_{L J} \approx 1.0$. Function $(7)$ is plotted in figure 2 by a dotted curve.

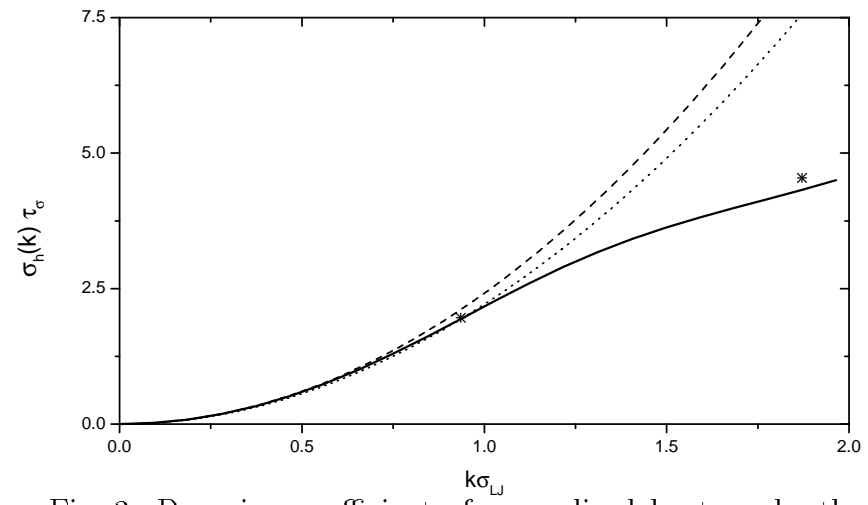

Fig. 3. Damping coefficient of generalized heat mode: the results of nine-mode approximation (solid curve), hydrodynamic behaviour (dashed line), mode-coupling prediction (dotted curve) and the results of five-mode approximation for $k / k_{\min }=1,2$ (stars), respectively.

In a similar way for fitting the generalized heat mode in a wider range of $k$ the expression

$$
\sigma_{h}^{a}(k)=D k^{2}-a_{h} k^{5 / 2}+b_{h} k^{11 / 4},
$$

with one adjustable parameter $b_{h}$ has been used. The quantities $D$ and $a_{h}$ were calculated from Eqs. (5) and (6). In this case we found that $b_{h}$ is much smaller in comparison with $b_{s}\left(b_{h}=-0.05\right)$, so that even the first two terms in $(8)$ describe well the behaviour of generalized heat mode up to $k \sigma_{L J} \approx 1.0$.

In Ref. [16] it was found that the $\mathrm{MC}$-like behaviour for the ratio of damping coefficients $\sigma_{s}(k) / \sigma_{h}(k)$ could be observed in much larger range of $k$ comparing with the functions $\sigma_{s}(k)$ and $\sigma_{h}(k)$. The $k$-dependence of $\sigma_{s}(k) / \sigma_{h}(k)$ is shown in figure 4 .

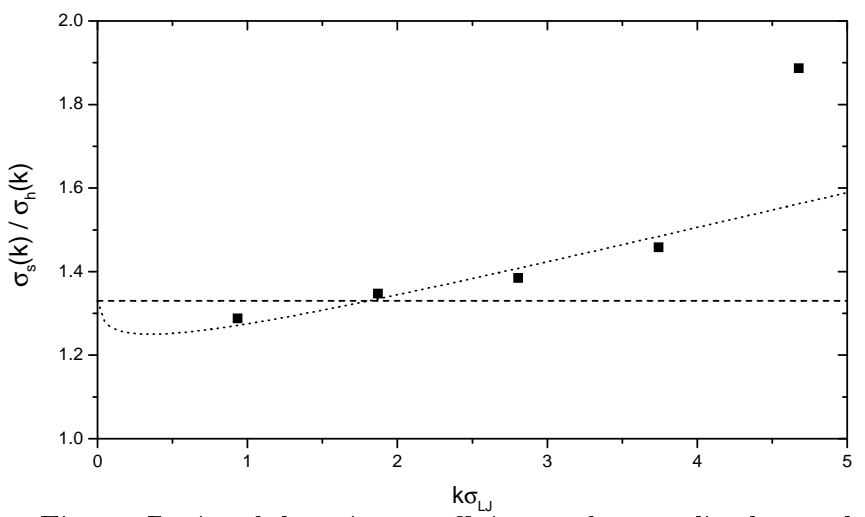

Fig. 4. Ratio of damping coefficients of generalized sound and heat modes: the results of nine-mode approximation for $k / k_{\min }=1,2,3,4,5$ (solid boxes), hydrodynamic behaviour (dashed line), and mode-coupling prediction (dotted curve).

The hydrodynamic value $\sigma_{s}(k) /\left.\sigma_{h}(k)\right|_{k \rightarrow 0}=\Gamma / D$ is plotted by a dashed line. From Eqs. (7) and (8) one has

$$
\frac{\sigma_{s}^{a}(k)}{\sigma_{h}^{a}(k)}=\frac{\Gamma}{D}-\delta_{1} k^{1 / 2}+\delta_{2} k^{3 / 4},
$$

where

$$
\delta_{1}=\frac{\Gamma}{D}\left(\frac{a_{s}}{\Gamma}-\frac{a_{h}}{D}\right)
$$

and

$$
\delta_{2}=\frac{\Gamma}{D}\left(\frac{b_{s}}{\Gamma}-\frac{b_{h}}{D}\right) .
$$

Using the estimations obtained above one gets $\delta_{1}=0.40$ and $\delta_{1}=0.35$. It is seen in figure 4 that the approximated result (9) is very close to $\sigma_{s}(k) / \sigma_{h}(k)$ up to $k \sigma_{L J}=3.5$ that is more than twice as large as the results found for $\omega_{s}(k), \sigma_{s}(k)$ and $\sigma_{h}(k)$ separately (see figures 2 and 3 ). The reason for such a behaviour is not quite clear. One may suppose only that the higher order MC contributions to $\sigma_{s}(k) / \sigma_{h}(k)$ are mutually canceled in contrary to the case of single damping coefficients $\sigma_{s}(k)$ and $\sigma_{h}(k)$. We note also that as is seen in figure 4 the deviation from $k$-dependence given by two first terms in (9) is already observed starting from extremely small values of $k$ $\left(k \sigma_{L J} \approx 0.05\right)$. This is in agreement with the conclusion of Ref. [19] and explains the $k$-dependence of an effective MC parameter $a_{s}(k)$ found in this paper. 


\section{CONCLUSION}

Summarizing the results obtained we conclude that:

(i) the MC effects for the generalized collective modes can be correctly described within the GCM approach;

(ii) the value of the MC parameter $a_{s}=0.98$ correlates well with the result $a_{s}=0.94$ obtained in Ref. [19] using the MC theory in nonlocal thermodynamic approximation;

(iii) comparing the data shown in figure 1,2, and 3 for five- and nine-mode approximations it is seen that the $\mathrm{MC}$-like behaviour is already reproduced even in lower mode approximation;

(iv) the higher order corrections $\left(b_{s}\right.$ and $\left.b_{h}\right)$ have been calculated by fitting the MC expressions to our data for the generalized hydrodynamic modes. Of course, it is interesting to obtain these quantities with the help of a more rigorous treatment. However, such calculations are very complex and require the knowledge of higher order distribution functions. We note that the simplification, usually applied in the mode-coupling theories by decomposing of the higher order correlation functions into the products of pair correlations can produce the results with an uncontrolled error.

(v) it should be stressed that the description of modecoupling effects can be considered as an additional test for any theory considered as the generalized hydrodynamic theory.

\section{ACKNOWLEDGMENTS}

This study is supported in part by the Fonds für Förderung der wissenschaftlichen Forschung under Project P12422 TPH. It is a pleasure to thank Prof. I.M. de Schepper for fruitful discussion regarding the applications of the GCM approach.
[1] J. P. Boon, S. Yip, Molecular hydrodynamics (McGrawHill Inc., New-York, 1980).

[2] U. Balucani, M. Zoppi, Dynamics of the liquid state (Clarendon Press, Oxford, 1994).

[3] B. Kamgar-Parsi, E. G. D. Cohen, I. M. de Schepper, Phys. Rev. A 35, 4781 (1987).

[4] I. M. de Schepper, E. G. D. Cohen, C. Bruin, J. C. van Rijs, W. Montrooij, L. A. Graaf, Phys. Rev. A 38, 271 (1988).

[5] I. M. Mryglod, I. P. Omelyan, M. V. Tokarchuk, Mol. Phys., 84, 235 (1995).

[6] I. M. Mryglod, I. P. Omelyan, Phys. Lett. A 205, 401 (1995).

[7] I. M. Mryglod, I. P. Omelyan, Mol. Phys. 90, 91 (1997).

[8] I. M. Mryglod, I. P. Omelyan, Mol. Phys. 91, 1005 (1997).

[9] I. M. Mryglod, I. P. Omelyan, Mol. Phys. 92, 913 (1997).
[10] I. M. Mryglod, Cond. Matt. Phys. (Lviv) 1, 753 (1998).

[11] K. Kawasaki, Ann. Phys., 61, 1 (1970).

[12] M. H. Ernst, J. R. Dorfman, J. Stat. Phys. 12, 311 (1975).

[13] A. F. Andreev, Zh. Eksp. Teor. Fiz. 75, 1132 (1978).

[14] A. F. Andreev, Zh. Eksp. Teor. Fiz. 78, 2064 (1980).

[15] V. G. Morozov, Physica A 117, 511 (1983).

[16] I. M. de Schepper, P. Verkerk, A. A. van Well, L. A. de Graaf, Phys. Lett. A 104, 29 (1984).

[17] S. V. Peletminskii, A. I. Sokolovskii, Ukr. Fiz. Zh. 37, 1521 (1992).

[18] S. V. Peletminskii, A. I. Sokolovskii, Ukr. Fiz. Zh. 37, 1702 (1992).

[19] V. V. Ignatyuk, M. V. Tokarchuk, preprint ICMP-9627U, (1996).

[20] The parameters $\sigma_{L J}$ and $\epsilon_{L J}$ denote the energy and space scales of Lennard-Jones potential.

\title{
ПРОЯВ ВЗАЕМОДЇ МОД У СПЕКТРАХ УЗАГАЛЬНЕНИХ ГІДРОДИНА МІЧНИХ ЗБУДЖЕНЬ Л ЕННАРД-ДЖОНСІВСЬКОЇ РІДИНИ
}

\author{
I. М. Мриглод \\ Інститут конденсованих систем НАН Украйни, \\ вул. Свениіцького, 1, Львік, 290011, Украӥна \\ E-mail: mryglod@icmp.lviv.ua
}

\begin{abstract}
Проаналізовано ефекти взаємодії мод у поведінці узагальнених гідродинамічних збуджень. Спектр колективних мод леннард-джонсівської рідини був розрахований [Phys. Lett. А 205 (1995) 401] y дев'ятимодовому наближенні методу узагальнених колективних мод. Показано, що отримані результати повністю узгоджуються з передбаченнями теорії взаємодіючих мод. Розраховано параметри взаємодії мод, які описують неаналітичні вклади до спектрів узагальнених гідродинамічних збуджень. Обговорено також зв'язок мі ж обома розглянутими теоріями.
\end{abstract}

